\title{
Dynamic Value Engineering Method Optimizing the Risk on Real Time Operating System
}

\author{
Dr Prashant Kumar Patra ${ }^{1}$, Padma Lochan Pradhan ${ }^{2}$ \\ ${ }^{1}$ Dept. of CSE, College of Engineering \& Technology, BPUT, Bhubaneswar-751003, Orissa, India. \\ ${ }^{2}$ Dept. of CSE, Central Institute of Technology, Raipur, CG, India. \\ e-mail: citrprcs@rediffmail.com.
}

\begin{abstract}
The value engineering is the umbrella of the many more sub-system like quality assurance, quality control, quality function design and development for manufacturability. The system engineering \& value engineering is two part of the coin. The value engineering is the high level of technology management for every aspect of engineering fields. The value engineering is the high utilization of System Product (i.e. Processor, Memory \& Encryption key), Services, Business and Resources at minimal cost. The high end operating system providing highest services at optimal cost \& time. The value engineering provides the maximum performance, accountability, reliability, integrity and availability of processor, memory, encryption key and other inter dependency sub-components. The value engineering is the ratio of the maximum functionality of individual components to the optimal cost. The VE is directly proportional to performance of individual components and inversely proportional to the minimal cost. The VE is directly proportional to the risk assessment. The VE maximize the business throughput \& decision process mean while minimize the risk and down time. We have to develop the dynamic value engineering method for risk optimization over a complex real time operating system.
\end{abstract}

Keywords: Value Engineering, Product Specification, Business Specification, Encryption, Processor, Memory, Value Method, High Availability

\section{Introduction}

In the present age, the technology management can be defined as the integrated planning, designing, organizing, operation and control of product \& services at minimal cost. The technology management improves the value engineering in a systematic \& continuous manner. The high performance of individual components at minimum cost will be high utilize at lowest cost, then improve the productivity of services and satisfy to the risk mgmt system. It is a systematic \& continuous process require for high productivity with low cost. [1], [6-7]

Product $(P, M, E, C, A)$

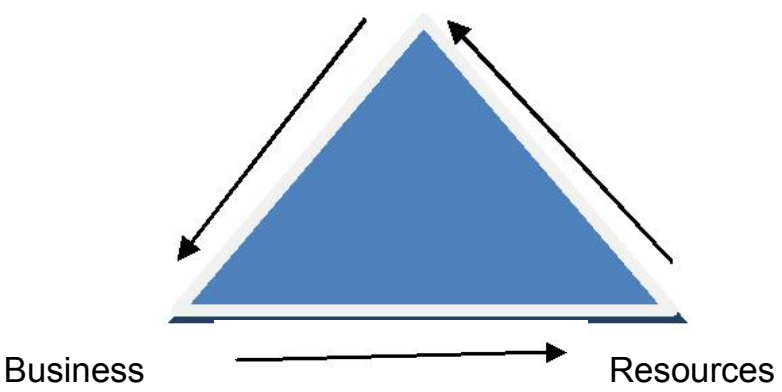

Figure 1. PBR Optimize the PME

The value engineering (VE) is a systematic approach to improve the value of goods or products and services by using real time experimental method. The value can therefore be increased by either improving the functionality and reducing the cost or both simultaneously. The value engineering is specifically establishing and maintaining cost-effective value 
engineering procedures and process in a systematic way. The value engineering is based on system engineering. The value engineering concept can be develop by combining system engineering (SE), reliability engineering (RE) and security engineering (SC). [3], [12].

The value methodology (VM) is a dynamic, systematic and structured approach, improves projects, products and processes. The VM is used to analyze manufacturing products and processes define, design, develop the projects, business and administrative processes. The VM helps to achieve balance among required functions, performance, quality, reliability, scalability, high availability, safety and scope with the cost and other resources necessary to accomplish those requirements. The proper balance results in the maximum value for the project. [3], [12].

\subsection{Value Engineering: Value $=$ Function $/$ Cost}

- Value is the reliable performance of functions to meet customer needs (Requirement Analysis) at the lowest overall cost.

- Function is the natural or characteristic action and reaction (behaviors and characteristics of components) performed by a product or service.

- Cost is the expenditure necessary to produce a project, goods, service, process and structure.

When the system engineering \& value engineering both meet each other, the risk assessment definite would be optimize at lowest cost. The risk assessment is the first process of the risk management methodology. Organizations use risk management to determine the extent of the potential threat and the risk associated with an IT system and sub system throughout its life cycle as the steps follow. The output of this process helps to identify appropriate controls (Preventive control, detective control, \& corrective control) for reducing or eliminating risk during the risk mitigation process, as discussed in proposed VE method. Risk is a function of the likelihood of a given threat-source's exercising a particular potential vulnerability, and the resulting impact of that adverse event on the organization. To determine the likelihood of a future adverse event, threats to an IT system must be analyzed in conjunction with the potential vulnerabilities and the controls in place for the IT system. Impact refers to the magnitude of harm that could be caused by a threat's exercise of vulnerability. The level of impact is governed by the potential mission impacts and in turn produces a relative value for the IT assets and resources affected (e.g., the criticality and sensitivity of the IT system components, devices and data [13], [18].

\subsection{Operating System}

The operating system is a collection of hardware, software \& application that manages system resources and provides common services for resources, program, application \& users. The operating system is an essential component of the system software (processor, memory, encryption, shell, file \& kernel) in computer system. The high level language (application programs) usually require an operating system to function. The Real time operating system is a multitasking, time sharing \& distributed operating system that aims at executing real-time applications. The real-time operating systems often use specialized scheduling algorithms so that they can achieve a deterministic nature of behavior. The main objective of real-time operating systems is their quick and predictable response to events. They have an event-driven or time-sharing design and often aspects of both. An event-driven system switches between tasks based on their priorities or external (resources) events while time-sharing operating systems switch tasks based on clock interrupts. [9-10], [15-16].

Operating system control is a step by step process of securely configuring a system to protect it against unauthorized access, while also taking steps to make the system more reliable and available. Generally anything that is done in the name of system. Preventive control ensures the system is secure, reliable and high available for high IT culture.

VE role in regards to Risk Mgmt:

- Communicate risk to top management by help of mobile message (mobichart).

- Organize \& understand variables affecting risk (High, Medium \& Low).

- Traditional cascading risk charts, risk matrix, risk register (Physical /Logical).

- Implement quantitative risk analysis, design \& implement.

- Assess current design state \& existing method and approach. (/var/adm/message) VE

IJEEI Vol. 2, No. 2, June 2014 : $101-110$ 
products provides decision making system.

- Removes or disable emotion element and objects from the system.

- Enables fact-based decisions making \& acquisition to top mgmt by help of mobile computing.

- Decision Analysis-builds consensus, defines alternatives, assigns priority and in a systematic manner.

- Define, design, develop and deployment and decide $\left(D^{\wedge} 4\right)$ in a systematic manner.

\section{Literature Survey [2], [9-10], [15-16]}

The technical literature survey in IS Security area is very, risk, critical \& tedious work to collect the actual data and analysis, investigation \& evidence in the real life system. It is one of the ongoing processes in a continuous manner. It is a very time consuming to investigate \& judge the information.

There are many text book \& reference books help to us to find out the real issue. The reference books like: Applied Cryptography by Bruce Schneier \& Cryptography \& Network Security by William Stalling is very much help full to expand our idea. The proposed model \& method is very helpful to application of cryptographic key management issue. The Sun Microsystem UNIX sun Solaris system administration guide: Vol 1 \& Vol 2. \& O' Reilly, Essential of System Administration is very helpful to collect the basic data [17], [18].

In our past experience, we observed on operating system as well as network server, there are many system parameters are defaults lacking behind over a larger application \& multiple resources and business on heterogeneous platform. There are many more issue arising like, memory, performance, bandwidth, network \& packets are slow down. This issue is highlighted in our problem statement, action plan \& proposed method.

We have to find out some method to make the more efficient, secure, high available \& robust high end operating system. No where develop the detail methods in Graphically as well as Mathematically about the risk management of the OS. There are many issues are not develop till now like: Risk Identification, Risk Analysis, Risk Mitigation \& risk results in the operating system level. We have to develop Risk Identification, Risk Analysis, Risk Mitigation in both analytical \& graphical way. There many documents are available in general sense of risk identifications, risk analysis, risk mitigation, but operating system level the classification \& categorization of risk is not available on today itself. We have to focus on the system specific like OS \& system software on VE, RM \& decision process. We can develop some optimization model, method and mechanism for risk mitigation based on technology survey. We have to consider the functionality of indivusal components of operating system, Product, Business \& Resources.

\subsection{Data Collection Based on Existing System Engineering: (Basic Data)}

There are number of system engineering preventive control methods developed as per requirement of the secure computing to achieve the highest level of business objective. UNIX file system have to be develop as per business requirement [2], [9-10], [15-16]. 
Table 1. Basic Data

\begin{tabular}{|c|c|c|c|}
\hline $\begin{array}{l}\mathrm{S} \\
\mathrm{N}\end{array}$ & $\begin{array}{l}\text { SYSTEM FILES INPUT } \\
\text { (Owner) }\end{array}$ & ACTION PLAN & REMARKS OUPUT \\
\hline 1 & /etc/system [PS] Product & Implement the kernel \& n-bit processer & $\begin{array}{l}\text { Can be improve the system } \\
\text { performance }\end{array}$ \\
\hline 2 & letc/hosts & $\begin{array}{l}\text { Develop the scripts:allow/disallow as per policy, } \\
\text { chmod000=/etc/hostnnmmxmdisallow }\end{array}$ & $\begin{array}{l}\text { Preventative control } \\
\text { Access control mechanism }\end{array}$ \\
\hline 3 & $\begin{array}{l}\text { letc/services [BS] } \\
\text { Services }\end{array}$ & $\begin{array}{l}\text { Disable the third parties services. Remove the ftp, http, } \\
\text { telnet, port no, printer, IP services. Those services are not } \\
\text { required. }\end{array}$ & Preventative control \\
\hline 4 & /use/bin/rsh, etc/ pam.conf & $\begin{array}{l}\text { Disable all remote services: chmod } 000 \text { /usr/bin/rsh, } \\
\text { rksh,rcp, ruser, rlogin, uptime. }\end{array}$ & Preventative control \\
\hline 5 & /var/adm/message & Date \& time stamp (events mgmt) & $\begin{array}{l}\text { Internal audit purpose Detective } \\
\text { control }\end{array}$ \\
\hline 6 & /etc/rc.conf script & $\begin{array}{l}\text { Run level script } \\
\text { Run level script have to develop as per requirement. } \\
\text { /etc/init.conf,rc2.d example:httpd_flags="NO" }\end{array}$ & Preventative control \\
\hline 7 & /etc/init.d & OS services, run level & Preventative control \\
\hline 8 & $\begin{array}{l}\text { etc/ssh/sshd_config } \\
\text { [PS] } \\
\text { Automated control }\end{array}$ & $\begin{array}{l}\text { Cryptography enable through ssh implementation AES: } 256 \\
\text { bits chipper. } \\
\text { chiper blowfish-CBC,aes256-CBC, aes256-chr.ssh-keygen } \\
\text {-b } 1024 \text {-f /etc/ssh_host_key -n " chmod - - - } \\
\text { letc/ssh/ssh_config }\end{array}$ & $\begin{array}{l}\text { Preventative control } n=1024, \\
2048,4096 \text { chmod } r \text { w } x \text { (i. e. } 4 \\
21 \text { ) - blank is nothing }\end{array}$ \\
\hline
\end{tabular}

\section{Existing Risk Assessment Method}

There are many preventive control has to design, developed and available in past \& present for risk assessment on Information System. The existing preventive controls are available for secure \& betterment of IT standard. There are six major area of the value engineering method as follows.

\subsection{Existing System}

The systematic and structural approach comes from the VM job plan. The VM job plan consists of six phases: [3], [9], [12].

1. Information Phase: Gather information to better understand the project definition. (Initial Stage)

2. Function Analysis Phase: Analyze the project to understand and clarify the required functions (RA).

3. Creative Phase: Generate ideas on all the possible ways to accomplish the required functions. (New product)

4. Evaluation Phase: Synthesize and analysis the ideas and concepts to select the feasible ideas for development into specific value improvement.

5. Development Phase: Select and prepare the best suggestions \& method alternative(s) for improving value of goods \& services.

6. Presentation Phase: Present the value recommendation to the project stakeholders/vendor, customer (services level).

The VM process produces the best results when applied by a multi-disciplined team with experience and expertise relative to the type of project to be studies in system engineering, reliable engineering \& security engineering.

\subsection{Existing Problem on Value Engineering (Technology, Engineering, Business)}

- The system engineering is a process which is not easy to accept under the normal conditions; the problem is commutative when it running several multiple jobs and applications simultaneously under complex IT infrastructure which using millions of user accessing the same piece of data and information in around the clock $(24 \times 7 \times 52)$.

When too many packets are present in the subnet, performances degrade and in this situation data/packet congestion is happening. In this way transmission error is happening on network (LAN-WAN). At the high end traffic, performance collapses completely and almost no packets are delivered. If there is insufficient memory to hold all of them, packet will be lost. If slow the processor can also cause the congestion. Similarly, the low bandwidth can also cause congestion. Therefore, the OS became hungering \& highly utilizing of CPU Times, system

IJEEI Vol. 2, No. 2, June 2014 : $101-110$ 
throughput became slow down, also slow down the network resources \& loss of communication system.

- There is no automatic protection, detection \& correction on the system components. There is no balance ratio among the Kernel, Processor, Memory, File System [Encryption Key] \& Time slot of the high end OS. The high level decision process is required to implement resources like kernel, processor and instruction level parallelism (SISD, SIMD, MISD, MIMD) \& high memory \& encryption key sizes for high end business. The high end technology would be match with high quality of business and decision.

\subsection{Research Questions}

Now a day increasing the third parties multiple users, applications of business, computer and communications system by IT industries has increased the risk of theft of proprietary data \& services. The operating system control \& audit is a primary method of protecting, detecting, correcting, operation and services of complex system resources.

- Increasing the millions of multiple as well as multipurpose users.

- Decreasing the performance, throughput, operation \& services over a complex infrastructure.

- Increasing the multiple layering \& distributed object oriented technology (SOA) to resolve the multiple requirements of Customers \& Clients, but mean while increasing the hacker, risk, uncertainty, theft \& unsecure.

- Increasing the hardware \& software capabilities ( $n$-th bits processor \& no of CPU, Memory).

- Increase the business \& technology, but technology \& business fully depends on political \& economic condition in around the globe.

\section{Methodology}

\section{Proposed Risk Assessment Method:}

There are many preventive method have to define, design, developed and deployment on a complex heterogeneous platform. The proposed preventive value engineering methods are available for secure, reliable \& high available for betterment of IT standard. There are seven points are mentioned on the development section as follows:

\subsection{Proposed SE Verification and Validation to Achieve our Objective to Gain the Value Engineering, which can be Optimize the Risk, Cost, Time and Maximize Throughput:}

The development of dynamic control algorithms, microprocessor (hardware) design system, and analysis of environmental systems also come within the purview of systems \& value engineering. The systems engineering encourages the use of tools and methods to better comprehend and manage the complexity in systems. Some examples of these tools can be define here as follow as: system model, system definition, behavior, architecture, optimization, reliability \& decision analysis.

In philosophically, thinking the concept of distributed object oriented system with the multi-disciplinary approach and method to system engineering is inherently complex since the characteristics \& behaviors of interaction among system components (objects) is not always immediately clear, defined and understand. The SE method is defining, designing \& developing the behavior \& characteristics such systems, subsystems (objects), resources and the interactions among them is one of the goals of systems engineering. In this way, the gap that exists among informal requirements from clients \& customer, users, operators, business requirements, resources (objects) requirements and technical specifications is successfully a long bridged.

We have to maintain the risk free environments in the hardware, software \& application level on the basis of the following data. We can update the SE parameters dynamically as per business $\&$ technology requirement any where $\&$ any time. That's why we are calling dynamic value engineering method.

\subsection{Define}

We have to define, design, develop and deployment the various method, model, mechanism, services and fix up majors automated system configuration to maintain residual risk. Meanwhile, we have to maintain the system control by applying automated method, model, 
mechanism $\left(\mathrm{M}^{\wedge} 3\right)$ \& tools on operating system level to optimize the risk and maximize the decision management to achieve the highest business objective. We have to define and initialize the Product, Business \& Resources to measure security domain for risk optimization and assessment.

SE: DECISION FACTOR OF THE PRODUCT SPECIFICATION: (INITIAL STAGE: PREVENTION MATRIX)

Table 2. Proposed PME Data

( DYNAMIC \& DERIVED DATA ):

ENCRYPTION

\begin{tabular}{|c|c|c|c|c|c|c|c|c|}
\hline $\mathrm{E}$ & 128 & 256 & 512 & & & $A=2^{\wedge} n$ & AES & $\overline{\mathrm{HA}}$ \\
\hline S & 512 & 1024 & 2048 & 4096 & 8192 & $S=2^{\wedge} n$ & $\mathrm{SSH}$ & HA \\
\hline $\mathrm{P}$ & 32 & 64 & 128 & 256 & 512 & $\mathrm{P}=2^{\wedge} \mathrm{n}$ & Processor & HA \\
\hline M & 16 & 32 & 64 & 128 & 256 & $\mathrm{M}=2^{\wedge} \mathrm{n}$ & Memory(GB) & HA \\
\hline $\mathrm{C}$ & $\mathrm{H}$ & $\mathrm{H}$ & $\mathrm{M}$ & $\mathrm{M}$ & $\mathrm{L}$ & $\mathrm{K}=2^{\wedge} \mathrm{n}$ & Control & $\mathrm{HA}$ \\
\hline
\end{tabular}

(L- LOW RISK, M-Medium RISK, H-HIGH RISK) (PC+DC+CC=C) [AES =k.1/R] FUZZ'S LAW FRAME WORK:

PRODUCT SPECIFICATION (INPUT) OF OPERATING SYSTEM:

The hardware designers have to decide the product specification to mitigate the risk

(i.e. target specification)

Let us consider;

PS= Product Specification, TS=Target Specification, BS=Business Specification, RS=Resource Specification

4.3. Algorithm: [Dynamic Product Development]

a. Decide the product specification (PS) as per business requirement(BS).(INPUT)

b. Analysis the Business \& Resource Specification

c. Select the metric elements should be dependable ( variables $P, M$ \& $E$ )

d. Select metric elements should be practicable ( variables $P, M \& E$ ).

e. Refine the product specification as per target specification(TS) (i.e. RM)

f. Establishing target specification(TS) (i.e. RM) as per business requirement.(OUTPUT)

g. Reflect on results and process.

BLACK BOX BLOCK DIAGRAM:

The Encryption $\operatorname{key}(E)$ should be define at the time of initial design stage for better system engineering as per top management decision. In this way, we can improve the value engineering.

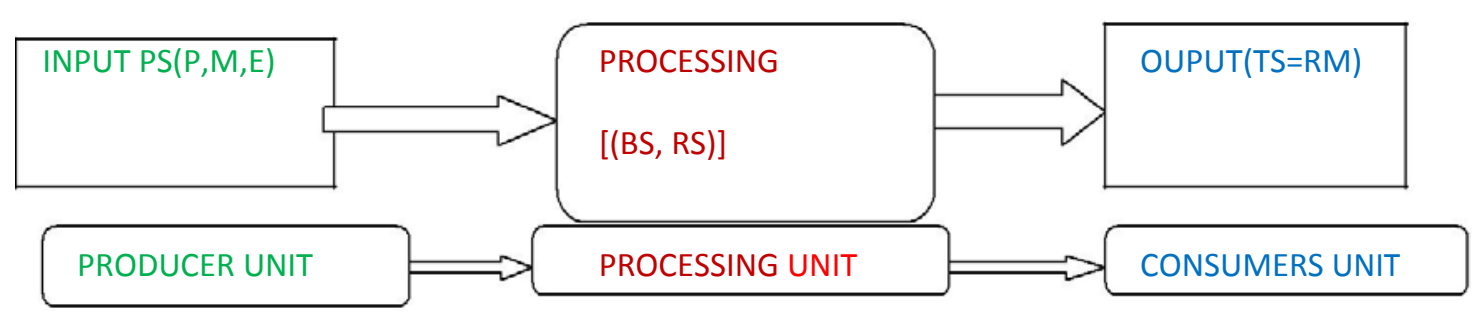

Figure 2. Black Box Diagram

\subsection{Design Stage}

We have to design the high reliable, scalable, security and highly available architecture to run the complex business on complex heterogeneous IT infrastructure to meet over the multi Relation, Function, Operation \& Service Level. [6-7] on the (PS)

We can planning, analysis and design of the following these two directed graph based

IJEEI Vol. 2, No. 2, June $2014: 101-110$ 


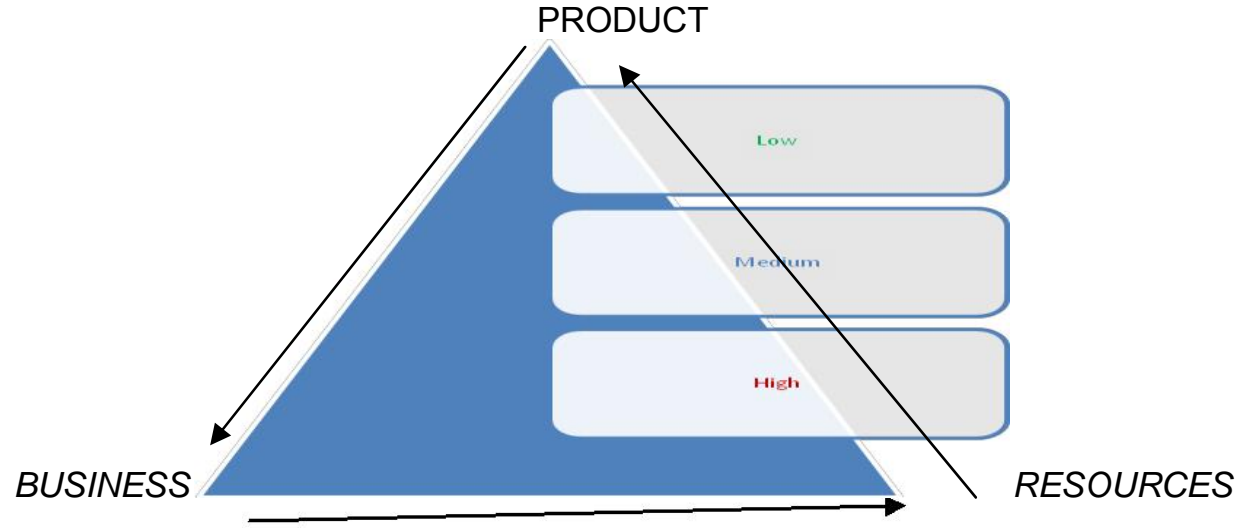

Figure 3 (a)

Associative Low: $(P \cup B) \cup R=P \cup(B \cup R),(P \cap B) \cap R=P \cap(B \cap R)$

Distributive Low: $P \cup(B \cap R)=(P \cup B) \cap(P \cup R), P \cap(B \cup R)=(P \cap B) \cup(P \cap R)$

\section{ENCRYPTION}

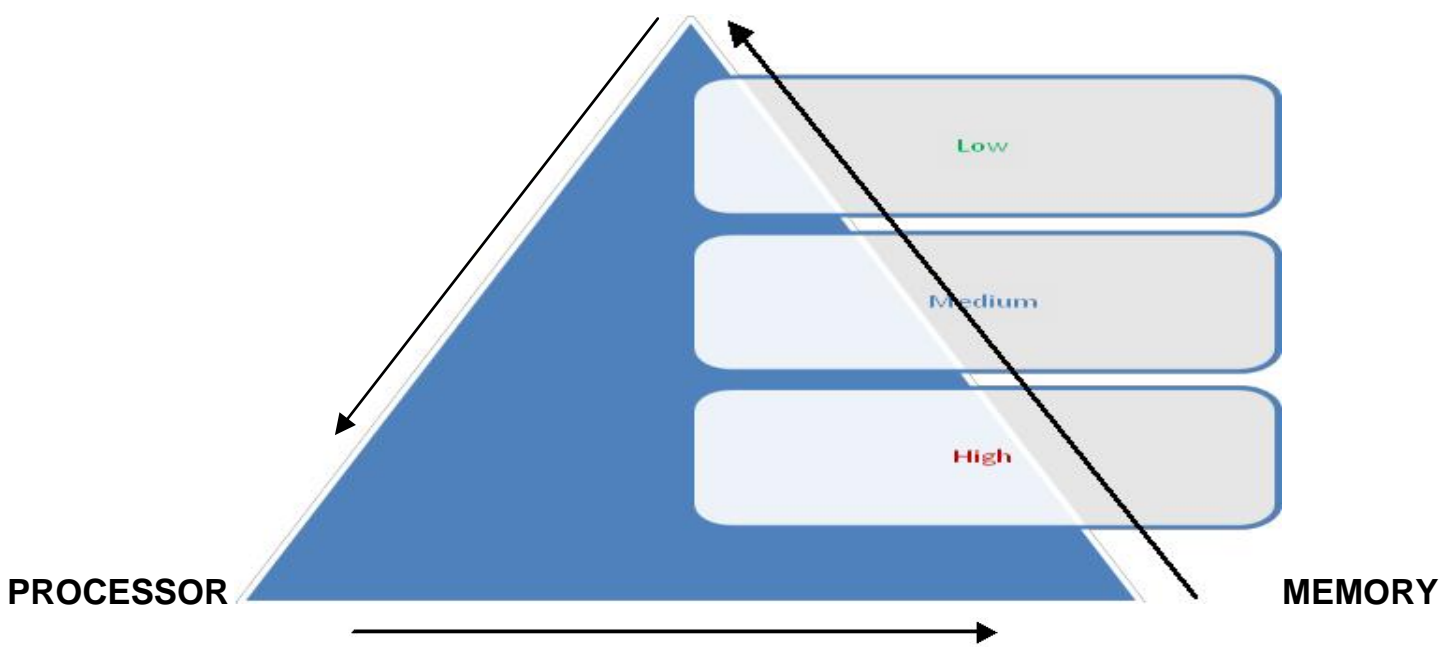

Fig:3 (b)

Associative Low: $(E \cup P) \cup M=E \cup(P \cup M),(E \cap P) \cap M=M \cap(P \cap M)$

Distributive Low: $E \cup(P \cap M)=(E \cup P) \cap(E \cup P), E \cap(P D \cup M)=(E \cap P) \cup(E \cap M)$

\subsection{Development}

We have to go forward to finding alternate optimization process on specification, process, engineering \& services for risk optimization based on operating system components $(P, M$ \& E). This scalable complex composion model definitely will be resolve our risk and security issue on complex real time system for multiple client application, business and resources available for multi location, vendor, customer on any time around the clock. PROPOSED METHOD OF OPTIMIZATION ON OPERATING SYSTEM RISK(GREEDY METHOD )

Our abstract is fully satisfied as theoretically, practically, analytically and graphically and assumption based data $\&$ attributes defined in this method. The value engineering is the ratio of functionalities of individual components (objects) to the optimal cost. Mathematical Deduction as follows: 
Table 3. Proposed Data

\begin{tabular}{|c|c|c|c|}
\hline SN & Equations & Descriptions & Remarks \\
\hline 01 & $\begin{array}{l}\text { VE }=F(P, M, E, C, A) / \\
\text { Optimal Cost }\end{array}$ & $\begin{array}{l}\text { Equation is satisfying to the operating } \\
\text { system. It is indicating characteristics } \\
\text { \& behavior of the system. } \\
\text { PRIMARY DECISION }\end{array}$ & $\begin{array}{l}\text { System Engineering: Improving the } \\
\text { performance of individual elements } \\
\text { Processor, Memory, Encryption key and } \\
\text { Availability (P, M, E, C, A) with respect to } \\
\text { optimal cost. } \\
\text { PRIMARY RISK ASSESSMENT }\end{array}$ \\
\hline 02 & $\begin{array}{l}\text { VE }=F(P S, B S, R S) / \text { Minimal } \\
\text { cost. } \\
\text { As per Algorithm. }\end{array}$ & $\begin{array}{l}\text { Where: PS: Product Specification, BS: } \\
\text { Business Specification, Resource } \\
\text { Specification \& TS: Target } \\
\text { Specification. } \\
\text { PRIMARY DECISION }\end{array}$ & $\begin{array}{l}\text { It will be help to define, design, } \\
\text { development \& implementation stage. } \\
\text { Encryption key should be added into design } \\
\text { phase, then cost \& time will be optimize. } \\
\text { Dynamic product development satisfying } \\
\text { the automated control. } \\
\text { PRIMARY RISK ASSESSMENT }\end{array}$ \\
\hline 03 & $\begin{array}{l}\text { VE = F ( P, S, O, M )/ Optimal } \\
\text { Cost }\end{array}$ & $\begin{array}{l}\text { Where: P: Product, S: Services, O: } \\
\text { Operation, M: Maintenance } \\
\text { SECONDARY DECISION }\end{array}$ & $\begin{array}{l}\text { Improving the product, services, operation } \\
\text { \& maintenance at minimum cost. } \\
\text { SECONDARY RISK ASSESSMENT }\end{array}$ \\
\hline 04 & $R=k \cdot 1 / C, C=k . S$ & $\begin{array}{l}\text { R-Risk, S- Standard, C-Control, k-prop } \\
\text { constant } \\
\text { SECONDARY DECISION }\end{array}$ & $\begin{array}{l}\text { Improve the quality, value \& reliability and } \\
\text { std. of system engineering. } \\
\text { SECONDARY RISK ASSESSMENT }\end{array}$ \\
\hline 05 & $\begin{array}{l}C=P+D+C \\
V E=F(P, D, C) / M i n i m a l ~ c o s t \\
V E=F(T C) / M C\end{array}$ & $\begin{array}{l}\text { P-Prevention, D-detection, C- } \\
\text { Correction. TC: Total Control, MC: Min } \\
\text { Cost. SECONDARY DECISION }\end{array}$ & $\begin{array}{l}\text { Maximize the prevention, detection \& } \\
\text { correction at law cost. } \\
\text { SECONDARY RISK ASSESSMENT }\end{array}$ \\
\hline 06 & $\mathrm{VE}=\mathrm{k} \cdot \mathrm{RA}$ & $\begin{array}{l}\text { Risk Assessment, k:Prop. Constant. } \\
\text { SECONDARY DECISION }\end{array}$ & $\begin{array}{l}\text { Optimize the risk } \\
\text { SECONDARY RISK ASSESSMENT }\end{array}$ \\
\hline 07 & $\begin{array}{l}\sum R M=\sum(V E+S E+R E+S E),(V E \\
U S E U R E U S C)=\sum R M\end{array}$ & $\begin{array}{l}\text { Composion of all four. } \\
\text { TOTAL DECISION }\end{array}$ & $\begin{array}{l}\text { Union of All. Satisfying to SC, RE, SE \& } \\
\text { VE. TOTAL RISK ASSESSMENT. }\end{array}$ \\
\hline
\end{tabular}

\subsection{Dynamic Composition Model Maximize Value Engineerng and Minimize the Risk}

MODEL \& MECHANISM

TECHNICAL MODEL OF Specification (INPUT)

BUSINESS MODEL OF Specification

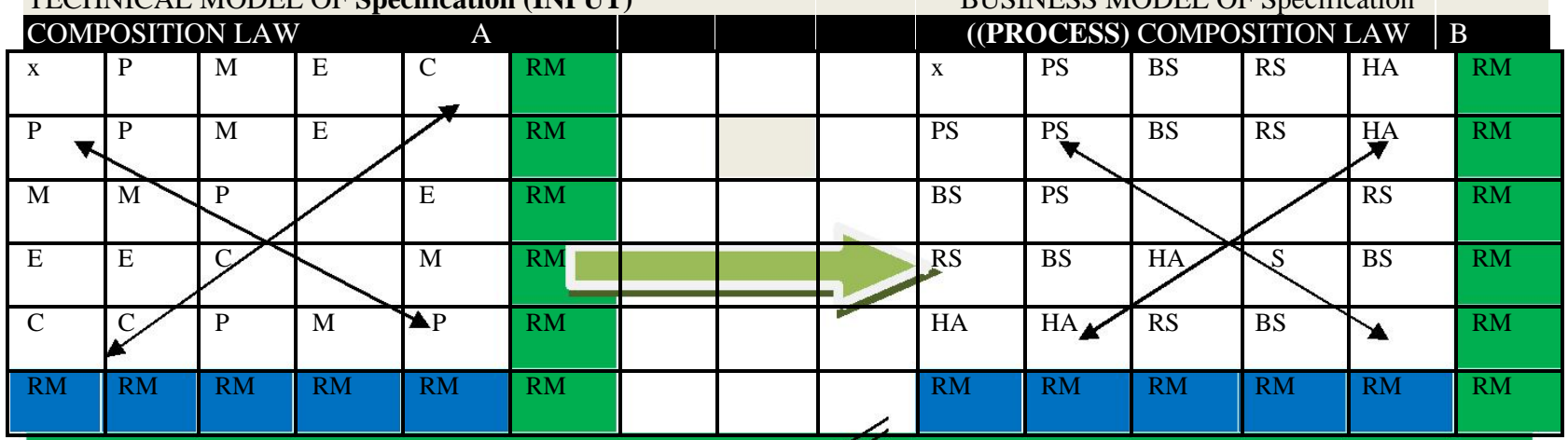

Both diagonal objects are not meeting our Objective

Where C:- Control, HA :- High Availability

ENGINEERING MODEL OF Specification. (PROCESS) COMPOSITION LAW

\begin{tabular}{|c|c|c|c|c|c|}
\hline $\mathrm{x}$ & VE & $\mathrm{SE}$ & $\mathrm{RE}$ & $\mathrm{SC}$ & $\mathrm{RM}$ \\
\hline VE & & SE & $\mathrm{RE}$ & & $\mathrm{RM}$ \\
\hline$\overline{\mathrm{SE}}$ & VE & & & $\mathrm{RE}$ & $\mathrm{RM}$ \\
\hline $\mathrm{RE}$ & $\mathrm{RE}$ & SC & & SE & $\mathrm{RM}$ \\
\hline $\mathrm{SC}$ & $\mathrm{SC}$ & $\mathrm{RE}$ & $\mathrm{SE}$ & $\mathrm{VE}$ & $\overline{\mathrm{RM}}$ \\
\hline $\mathrm{RM}$ & $\mathrm{RM}$ & $\mathrm{RM}$ & $\mathrm{RM}$ & $\mathrm{RM}$ & $\mathrm{RM}$ \\
\hline
\end{tabular}

Both diagonal objects are not meeting our Engineering
Both diagonal objects are not meeting our Purpose.

SERVICE MODEL OF Specification(OUTPUT)

\begin{tabular}{|l|l|l|l|l|l|}
\hline \multicolumn{6}{|c|}{ COMPOSITION LAW } \\
\hline P & P & S & O & M & RM \\
\hline S & S & S & O & M & RM \\
\hline & O & M & & S & RM \\
\hline M & M & O & S & & RM \\
\hline RM & RM & RM & RM & RM & RM \\
\hline
\end{tabular}

Both diagonal objects are not giving any Output ( TS)

IJEEI Vol. 2, No. 2, June 2014 : 101 - 110 

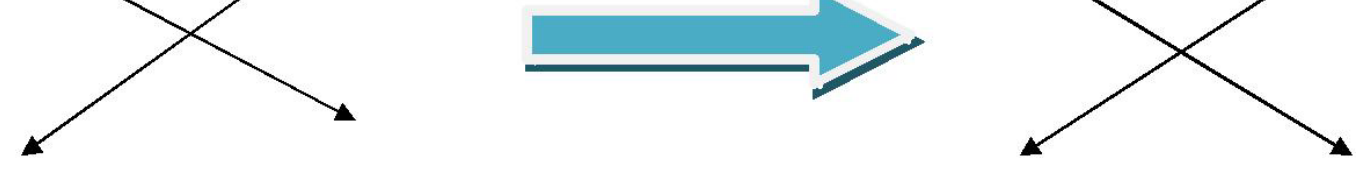

The Raw and Column total are meeting our Target Specificication, Output, Objective \& Purpose on multiple Product, Business, Resources \& Applications over a heterogeneous complex platform.

\section{Results \& Discussion (Services)}

We have to gain the maximum objective as per mix culture of the theoretical as well as practical services over a complex real time operating system.

- Maximize the protection, detection, correction, operation \& services at optimal cost and time.

- Maximize the (functionalities) performance, integration, availability, reliability at optimal cost.

- Maximum utilization of product, business, resources at minimal cost at right time in right way.

The symmetrical object are not meeting the risk mitigation, but the anti-symmetrical objects only resolving our purpose.

In this ways, we can improve the business and optimize the resource \& technology cost, mean while improve the performance of product \& services, Therefore, the value engineering ultimately \& automatically optimize the risk mgmt system and help to the decision making to the top mgmt. It is not only value engineering but also satisfying to the reliability engineering as well as security engineering.

\section{Conclusion}

In the final value analysis of the product, value engineering is not only beneficial, but also essential because of:

- The functionality of the project (PM) is often improved as well as producing tremendous savings, both initial and Life-Cycle Cost. (Block Diagram)

- A second look at the design produced by the (designer \& reengineering (Table 3) architect and engineers gives the assurance that all reasonable alternatives have been explored. [PS(P, M, E)]

- The cost estimates, reduction and scope statements are checked thoroughly assuring that nothing has been omitted or underestimated. (Cost optimization)

- Assures that the best value will be obtained over the life of the building. (Define, design, development, deployment \& decision (TS).

An automated system engineering used to be develop on the decision criteria when it is important to secure as much as possible of what is wanted from each components (objects) or unit of the resource used. The resource may be money, space, time, man, machine, material, energy, market, method and so on. The system is unique in that it effectively uses both knowledge, creativity and provides step-by-step techniques for maximizing the benefits from each component. It promotes development of alternatives suitable for the future as well as the present. This is accomplished by identifying and studying each function that is wanted by the customers and clients or user, then applying knowledge and creativity to achieve the desired functions. Resources are converted into costs to achieve direct, meaningful comparisons. By using this methods of value engineering, we can achieve the $30 \%$ to $40 \%$ reduction in the required resources often results.

The dynamic value engineering methodology helps to the any organization the more effectively in local, national and international markets at any time and any place around the clock follows:

- Minimizing costs \& risk.

- $\quad$ Maximize profits, ROI \& TCO.

- Improving functionality, quality \& decision (TQM).

- Maximizing Production, Sales, Market, Share \& generate the Capital.

- Optimizing time \& Maximize the utilization of Man, Machine, Material, Market, Money \& Method $\left(M^{\wedge} 5\right)$.

- Solving multiple problems at right time with optimal cost.

- Utilizing overall resources more effectively at right time and right place. 


\section{VE is a Better to Butter:}

- The changes are executed at the initial stages only (PS). We are already defined in BLOCK DIAGRAM.

- It requires specific technical knowledge. [SE] Table: $2 \& 3$

The value engineering provides accountability for individual's functionality of each components of the real time operating system over a application, system software, server and network. This accountability is accomplished through optimization model and mechanisms that require, accountability, availability, reliability \& integrity of the automated optimization control functions which is call Security Engineering, Reliability Engineering \& System Engineering. That's why this value engineering is practically and theoretically working as process of risk optimization and decision making criteria on technology management system in around the globe.

\section{Recommendations}

\section{Future Advancement of this Work:}

- We have to keep balance \& managing work load ratio among the Product, Business, Resources over a multiple application, network and infrastructure by applying value engineering model, method \& mechanism.

- We have to develop the distributed object oriented value engineering methods for multiple business, product \& resources over a multiple applications \& heterogeneous platform as per customer requirements.

\section{References}

[1] Bernard, Kolman. Discrete Mathematical Structures. New Delhi, India: Person Education India. (PHI). 2007.

[2] Bruce, Schneier. Applied Cryptography, New Delhi, India: Wiley Publishing Inc. 1996.

[3] B Mohadevan. Operation Management, New Delhi India: Person Education India. PHI 2008.

[4] CISA Review Mannual. ISACA, USA. 2003.

[5] Coriolis. CISSP Exam Cram, Coriolis Group Books, New Delhi, India: Dreamatech. 2002.

[6] Edgar G. Discrete Mathematics with Graph Theory. New Delhi, India: Person India. (PHI). 2007.

[7] Joe L Matt. Discrete Mathematics for Scientist and Mathematician. New Delhi, India: Person Education India. (PHI). 2008.

[8] John B Kramer. The CISA Prep Guide, New Delhi, India: Wiley Publishing Inc. 2003.

[9] Hwang, Kai. Advance Computer Architecture. New Delhi, India: Tata McGraw Hill. 2008.

[10] O' Reilly. Essential of System Administration. O' Reilly Media: USA. 1995.

[11] Pressman, Software Engineering. New Delhi, India: Tata McGraw Hill. 2001.

[12] Richard B Chase, Robert, Nicholas, Nitin. Operations Management. New Delhi, India: Tata McGraw Hill. 2006.

[13] Shon, Harrish. CISSP Exam study guide, New Delhi, India: Dreamtech. 2002.

[14] Shon, Harrish. Security Mgmt Practices. New Delhi, India: Wiley Publishing Inc. 2002.

[15] Sumitabh, Das. UNIX System V UNIX Concept \& Application. Delhi, India: Tata McGraw Hill. 2009.

[16] Sun-Microsystem. UNIX Sun Solaris system administration. USA. 2002.

[17] William, Stalling. Cryptography and Network Security. New Delhi, India: Person India. 2006.

[18] Weber, Ron. Information System Control \& Audit. New Delhi, India: Person Education India. (PHI). 2002. 